\title{
Accurate measurement of the longitudinal thermal conductivity and volumetric heat capacity of single carbon fibers with the $3 \omega$ method
}

\author{
Ketaki Mishra $^{\text {(a) }}{ }^{*}$, Bertrand Garnier ${ }^{\text {(a) }}$, Steven Le Corre ${ }^{\text {(a) }}$, Nicolas Boyard ${ }^{(a)}$
}

a CNRS, LTeN UMR 6607, Université de Nantes, Rue Christian Pauc, 44306 Nantes Cedex 3, France.

Corresponding author : ketaki.mishra@univ-nantes.fr, 0240683180

\begin{abstract}
:
An experimental setup for $3 \omega$ method with a constant current source and two differential amplifiers was built to measure the thermal conductivity and the volumetric heat capacity of single polyacrylonitrile (PAN)-based carbon fiber. In complement to a well known analytical thermal model, a numerical one was developed that can check the validity of the analytical one and can also take into account the effect of convective heat loss on the measurements. A detailed sensitivity analysis of the unknown parameters was presented that would finally help in the better design of the setup for $3 \omega$ method. The tests were performed under vacuum and atmospheric pressure for chromel wire as a reference sample and under vacuum for two types of polyacrylonitrile-based carbon fiber. Detailed measurements were performed displaying the influence of convective loss and the thermal contact resistance between fiber and copper electrodes on the estimation of thermal properties of carbon fiber.
\end{abstract}

Keywords: $3 \omega$ method; thermal conductivity; carbon fibers; sensitivity analysis; thermal contact resistance.

\section{Introduction}

In the current engineering scenario, carbon fibers reinforced composites have found a great place in many high-performance industries such as aviation, automotive, textile, etc. With the advances in the material technology, many researchers focused their interest in finding out the effective thermal properties of the composite material at different scales starting from macro to micro. Although a lot of research work can be found at macroscale [1][2][3], less studies were dedicated to the estimation of thermal properties at microscale such as at the level of single fiber.

In the literature, some methods use a bundle of fibers to approximate the thermal properties of single fiber such as Angström method or Thermal potentiometer [4] or Laser flash method [5]. There are also a few techniques that work directly on single fibers such as AC calorimetry [6], T-type probe method [7], pulse laser-assisted thermal relaxation technique [8]. A brief detail of these techniques is presented in Table1. Some thermal conductivity values measured using these techniques are shown in Table 2 for different types of carbon fibers. These techniques mainly focus on the estimation of single thermal property such as either thermal conductivity or specific heat or thermal diffusivity during one experiment. Contrary to these techniques, the $3 \omega$ method is a strong benchmark that has the capability of measuring these thermal properties simultaneously. Furthermore, the applicability of the $3 \omega$ method is large, it was used for solids at micro[9] to macro scales[10], liquids and gases[11][12]. Less focus was given in adopting the $3 \omega$ method for measuring the thermal properties of single carbon fiber.

This technique consists in heating the sample with an alternating current at a frequency $\omega$. The current leads to temperature fluctuations at $2 \omega$, ultimately resulting in fluctuations of the voltage at both $\omega$ and $3 \omega$ as it will be discussed in details further. The variation of the $3 \omega$ voltage with the frequency contains the information about the 
thermal properties such as thermal conductivity and volumetric heat capacity of the sample. It is therefore important to eliminate the $1 \omega$ voltage by certain improvement in the setup such as a Wheatstone bridge [9] or an analog balance bridge circuit [13].

\begin{tabular}{|c|c|c|c|c|}
\hline Method & Heating Method & Temperature sensor & Sample Type & Reference \\
\hline Angstrom Method & $\begin{array}{l}\text { Constant DC current } \\
\text { heater (Joule Heating) }\end{array}$ & Thermocouples & Fiber Bundle & {$[4]$} \\
\hline Laser Flash Method & Short Laser Pulse & IR sensor & Fiber Bundle & {$[5]$} \\
\hline $\begin{array}{c}\text { Thermal } \\
\text { Potentiometer }\end{array}$ & $\begin{array}{c}\text { Constant DC heater } \\
\text { (Joule Heating) }\end{array}$ & Thermocouples & Fiber Bundle & {$[4]$} \\
\hline AC Calorimetery & Halogen Lamp & Thermocouple & Single Fiber & {$[6]$} \\
\hline $\begin{array}{l}\text { T-Type Probe } \\
\text { Method }\end{array}$ & $\begin{array}{c}\text { Constant DC heater } \\
\text { (Joule Heating) }\end{array}$ & Hot wire & Single Fiber & [7] \\
\hline $\begin{array}{l}\text { Periodic heating } \\
\text { technique }\end{array}$ & Argon Laser Beam & IR sensor & Single Fiber & {$[8]$} \\
\hline 3w Method & $\begin{array}{c}\text { AC power supply } \\
\text { heater (Joule Heating) }\end{array}$ & Sample dependent. & Single Fiber & [9] \\
\hline
\end{tabular}

Table 1 Literature survey on different methods used for thermal property measurement of carbon fiber

This not only increases the stability of the $3 \omega$ voltage but also increases the accuracy in the estimation of thermal properties [15]. In the literature, there are multiple experimental configurations based on the type of sources such as constant current [13] or constant voltage source [16]. The milestone research work was provided in the paper of $\mathrm{Lu}$ [9] where he discussed the simultaneous measurement of the thermal conductivity and volumetric heat capacity of single platinum wire and carbon nanotube bundles acting itself as heater and sensor. This paper provided the basic analytical thermal model that can enable the estimation of thermal properties and many detailed experimental tips while performing the $3 \omega$ method experiments under vacuum. It also dealt with radial heat losses due to radiation and convection by choosing proper approximation. None of the previous research showed the sensitivity of the measured voltage with respect to the thermal properties as function of the working frequency range that directly depends on the length of the sample used. The lack of sensitivity could explain the variation of measurement of thermal properties with change in the length for the same sample [13].

This paper is aimed at providing a detailed analysis of the implementation of a $3 \omega$ method for carbon fibers, giving design tips for a proper choice of sample dimensions and frequency range and quantifying the precision of the measurements. After presenting the principle and the analytical modeling of the $3 \omega$ method [9] applied to the measurement of the thermal properties for wires or fibers, we show a complementary numerical formulation of the heat transfer equation that enables to account for lateral heat losses. On the basis of those tools, a sensitivity analysis of the measured voltage with respect to the thermal conductivity and volumetric heat capacity over a wide frequency range $\left(10^{-2}-10^{4} \mathrm{~Hz}\right)$ is then presented. It highlights the importance of choosing a proper length of the sample with higher sensitivity that can ultimately improve the accuracy of the measurements. This leads to a proper experimental design which is detailed afterwards. Lastly, the thermal conductivity and volumetric heat capacity 
are measured for a chromel wire and two types of PAN-based carbon fibers and the effect of thermal contact resistance between sample and its holder is discussed.

\begin{tabular}{|c|c|c|c|c|c|c|}
\hline $\begin{array}{c}\text { Carbon } \\
\text { Fiber }\end{array}$ & $\begin{array}{c}\text { Commercial } \\
\text { Reference }\end{array}$ & $\begin{array}{c}\text { Thermal } \\
\text { Conductivity }\left(\mathbf{W m}^{-1} \mathbf{K}^{-1}\right)\end{array}$ & $\begin{array}{c}\text { Thermal } \\
\text { Diffusivity }\left(\mathbf{m m}^{2} \mathbf{s}^{-1}\right) \\
\end{array}$ & $\begin{array}{l}\text { Temperature } \\
\text { range }(\mathbf{K})\end{array}$ & Method & Reference \\
\hline \multirow{6}{*}{ PAN Based } & IM7 & - & 7.48 & $\mathrm{RT}^{*}$ & Laser Flash & [5] \\
\hline & M55J & - & 81.15 & RT & Laser Flash & [5] \\
\hline & UKY & - & 5.91 & RT & Laser Flash & [5] \\
\hline & PANEX 33 & - & $15-20$ & $850-1250$ & Periodic heating & [8] \\
\hline & AS4 & 6.5 & - & RT & $3 \omega$ Method & [10] \\
\hline & IM10 & 6.9 & - & $\mathrm{RT}$ & $3 \omega$ Method & [10] \\
\hline \multirow{3}{*}{ Pitch Based } & K1100 & - & 415.17 & $\mathrm{RT}$ & Laser Flash & [5] \\
\hline & P100 & - & $85-45$ & $750-1875$ & Periodic heating & [8] \\
\hline & - & 490 & - & RT & T-type & [7] \\
\hline $\begin{array}{l}\text { Rayon } \\
\text { Based }\end{array}$ & $\mathrm{TC} 2$ & $5-12.5$ & - & $850-1800$ & Periodic heating & [8] \\
\hline
\end{tabular}

Table 2 Longitudinal thermal conductivity and thermal diffusivity values of carbon fiber from literature. $\left({ }^{*} \mathrm{RT}=\right.$ Room temperature $)$

\section{Analytical Model}

The schematic diagram of the sample over the copper electrodes is depicted in Fig. 1. The fiber, assumed straight and of length $L$, is connected to two electrodes of constant temperature and submitted to an alternating current with a given frequency. The measurement of the voltage between the two electrodes over a certain range of frequencies is then used to identify the thermal properties, by using a thermal model, which can be either analytical or numerical.

This section provides a brief introduction of the analytical model for the $3 \omega$ voltage response of a self-heating fiber depending on the imposed frequency $(\omega)$. The model assumes that the specimen is a uniform cylinder placed between two heat sinks (copper electrode) with perfect contact at the joints (Fig. 1). An alternating electrical current $\left(I_{0} \sin (\omega t)\right)$ with a frequency $\omega$ passed through the sample that has an electrical resistance $R$ and length $L$.

Fig 1 Schematic diagram of the fiber sample with copper holders

$\mathrm{Lu}$ [9] has developed an analytical model for 1D heat conduction with a source term along the fiber that can predict the $3 \omega$ voltage through the sample. The partial differential equation for solving the corresponding heat conduction problem is as follows:

$$
\rho C_{p} \frac{\partial}{\partial t} T(x, t)-k \frac{\partial^{2}}{\partial x^{2}} T(x, t)=I_{0}^{2} \sin ^{2} \omega t\left[R_{0}\left(1+\alpha_{e}\left(T(x, t)-T_{0}\right)\right]\right.
$$

with the following boundary and initial conditions:

$$
\left\{\begin{array}{l}
T(0, t)=T_{0} \\
T(L, t)=T_{0} \\
T(x, 0)=T_{0}
\end{array}\right.
$$

where $\rho$ is the density, $C_{p}$ is the heat capacity, $k$ is the thermal conductivity, $\omega(2 \pi f)$ is the angular frequency, $f$ is the frequency, $T$ is the temperature of the sample at position $x$ and time $t, T_{0}$ is the ambient temperature, $I_{0}$ is 
the amplitude of the current, $R_{0}$ is the electrical resistance of the fiber at $T_{0}, \alpha_{e}$ is the temperature coefficient of this electrical resistance. By applying impulse theorem the temperature distribution through the sample can be derived as [9]:

$$
T(x, t)-T_{0}=\Delta_{0} \sum_{n=1}^{\infty} \frac{\left[1-(-1)^{n}\right]}{2 n^{3}} * \sin \left(\frac{n \pi x}{L}\right)\left[1-\left(\frac{\sin \left(2 \omega t+\phi_{n}\right)}{\sqrt{1+\cot ^{2} \phi_{n}}}\right)\right]
$$

where $\cot \phi_{n}=\frac{2 \omega \gamma}{n^{2}}, \gamma=\frac{\rho C_{p} L^{2}}{\pi^{2} k}$ is the thermal time constant for axial thermal transfer and $\Delta_{0}=\frac{2 I_{0}{ }^{2} R L}{\pi k S}$ is the maximum DC temperature accumulation at the center of the specimen. Eq. 3 is obtained under the following condition that enables the proposed truncation Eq.3 of the solution.

$$
\delta_{0}=\frac{I_{0}^{2} R^{\prime} L}{n^{2} \pi^{2} k S} \ll 1
$$

where $R^{\prime}=R_{0} \alpha_{e}$ and $S$ is the cross sectional area. The temperature fluctuating at a frequency of $2 \omega$ gives rise to a resistance fluctuation at the same frequency and can be expressed as:

$$
\delta R=R^{\prime} \Delta_{0} \sum_{n=1}^{\infty} \frac{\left[1-(-1)^{n}\right]^{2}}{2 \pi n^{4}}\left[1-\left(\frac{\sin \left(2 \omega t+\phi_{n}\right)}{\sqrt{1+\cot ^{2} \phi_{n}}}\right)\right]
$$

where $\phi_{n}$ is the value of the phase constant. The expression of resistance fluctuation can then be added to the initial resistance $R_{0}$ of the sample to obtain the total resistance $\left(R_{0}+\delta R\right)$. This value of total resistance is then multiplied with the applied alternating current $I_{0} \sin (\omega t)$ to obtain the voltage drop across the sample,

$$
V=I R=I\left(R_{0}+\delta R\right)=f(\omega)+g(3 \omega)
$$

The voltage has two components, one depending on frequency $\omega$ and the other one on $3 \omega$. The $3 \omega$ part contains the information about the thermal properties as it is the fluctuating temperature dependent part of the overall voltage drop. The average (Root Mean Square) voltage at $3 \omega$ frequency can be written as follows [9]:

$$
V_{3 \omega r m s} \approx-\frac{4 I_{r m s}^{3} L R R^{\prime}}{\pi^{4} k S \sqrt{1+(2 \omega \gamma)^{2}}}
$$

The RMS values of voltage at frequency $3 \omega\left(V_{3 \omega r m s}\right)$ is therefore a function of frequency and other parameters that are constants to be measured beforehand. Thus fitting this model with the experimental results enables to estimate the unknown parameters which are thermal conductivity and volumetric heat capacity of the sample under study. Successful implementation of this model obviously requires an accurate measurement of all the parameters governing Eq. 7 such as RMS current $\left(I_{r m s}\right)$, length $(L)$, temperature coefficient of resistance $\left(\alpha_{e}\right)$, cross sectional surface area of the sample $(S)$.

In the same work [9], the condition for neglecting the heat losses due to radiation is also given. It states that conductive power should be much higher than the power dissipated due to radiation.

$$
\delta_{1}=\frac{16 \epsilon \sigma T_{0}^{3} L^{2}}{\pi^{2} k D} \ll 1
$$

where $\epsilon$ is the fiber emissivity and $\sigma$ the Stefan Boltzmann constant and $D$ is the diameter of the sample under study. 


\section{Numerical model}

Previous researches on $3 \omega$ method were restrictive to experiments in a vacuum chamber. This motivated the development of a numerical model that could not only work under vacuum but also approximate the effect of convective losses. This section shows a brief description of the numerical model for estimating the $3 \omega$ response of one fiber in the configuration depicted in Fig. 1 after passing an alternating current. In the case of constant thermophysical parameters, the heat transfer phenomena can be described by the following 1D partial differential equation:

$\frac{\rho C_{p}}{k} \frac{\partial}{\partial t} T(x, t)-\frac{\partial^{2}}{\partial x^{2}} T(x, t)+m^{2}\left(T(x, t)-T_{o}\right)=\frac{Q}{k}$

where $m^{2}=4 h / k D$ with $h$ the heat transfer coefficient of air that surrounds the sample. $Q$ is the average volumetric heat source due to the alternating current. By choosing $\Delta T(x, t)=T(x, t)-T_{o}$, the above equation can be transformed to

$\frac{\rho C_{p}}{k} \frac{\partial}{\partial t} \Delta T(x, t)-\frac{\partial^{2}}{\partial x^{2}} \Delta T(x, t)+m^{2}(\Delta T(x, t))=\frac{Q}{k}$

Implementing one of the deductions from the analytical model that the temperature is fluctuating at $2 \omega$ frequency and so as the volumetric heat source $\left(2 R I_{r m s}^{2} / S L \sim f(2 \omega)\right)$, the terms $\Delta T$ and $Q$ can be sought in the following periodic form:

$\Delta T=\operatorname{Re}\left(\widetilde{T} e^{j 2 \omega t}\right), Q=\operatorname{Re}\left(\tilde{Q} e^{j 2 \omega t}\right)$

where $\widetilde{T}$ and $\widetilde{Q}$ are the complex expressions of the temperature drop and volumetric power respectively. Re designates the real part of a complex expression. Eq. 10 can be transformed by implementation of Eq. 11 to:

$\frac{\partial^{2} \tilde{T}}{\partial x^{2}}=-\frac{\tilde{Q}}{k}+\left(\frac{j 2 \omega \rho \mathrm{C}_{p}}{k}+m^{2}\right) \tilde{T}$

The spatial boundary conditions present in Eq. 2 can be transformed to Eq. 13

$$
\begin{cases}\tilde{T}=0, & x=0 \\ \tilde{T}=0, & x=L\end{cases}
$$

A 1D mesh representative of the sample was generated and a central finite difference approach was used to solve the Eq. 12 by implementation of the boundary conditions of Eq. 13 . The discrete matrix representation of the Eq. 12 and 13 is

$$
\left[\begin{array}{c}
\tilde{T}_{2} \\
\tilde{T}_{3} \\
\vdots \\
\vdots \\
\vdots \\
\tilde{T}_{N-1}
\end{array}\right]=\left[\begin{array}{cccccc}
\beta & \gamma & 0 & \ldots & \ldots & 0 \\
\gamma & \beta & \gamma & 0 & \ldots & 0 \\
0 & \gamma & \beta & \gamma & \ldots & 0 \\
\vdots & \vdots & \ddots & \ddots & \ddots & 0 \\
\vdots & \vdots & 0 & \ddots & \ddots & \ddots \\
0 & 0 & 0 & 0 & \gamma & \beta
\end{array}\right]^{-1}\left[\begin{array}{c}
\delta \\
\vdots \\
\vdots \\
\vdots \\
\delta
\end{array}\right] \text { with }\left\{\begin{array}{c}
\gamma=\frac{1}{\Delta x^{2}} \\
\beta=-\left[\frac{2}{\Delta x^{2}}+\frac{j 2 \omega \rho C_{p}}{k}+m^{2}\right] \\
\delta=-\frac{\tilde{Q}}{k}
\end{array}\right.
$$


The complex form of the temperature rise at all nodes $\left(\tilde{T}_{2}, \tilde{T}_{3} \ldots \widetilde{T}_{N-1}\right)$ can be estimated by solving this linear system. The modulus of the average of this temperature rises over all nodes $(N)$ gives the average longitudinal temperature rise $T_{a v}$ of the sample:

$$
T_{a v}=\left\|\sum_{1}^{N} \tilde{T}_{i}\right\| / N
$$

The overall voltage response measured in $3 \omega$ method is the modular $V_{3 \omega r m s}$ of the sample and thus the numerical $V_{3 \omega r m s}$ can be expressed as follows:

$$
\begin{aligned}
& V_{r m s}=I_{r m s}\left(R_{0}+R_{0} \alpha_{e} T_{a v}\right)=f(\omega)+g(3 \omega) \\
& V_{3 \omega r m s}=I_{r m s} R_{0} \alpha_{e} T_{a v}
\end{aligned}
$$

This step is repeated for estimating $V_{3 \omega r m s}$ for the desired frequencies. The effect of convective loss during the measurements under atmospheric condition can be approximated by implementation of heat transfer coefficient[17] in the term ' $m$ ' present in Eq. 14.

\section{Comparison between analytical and numerical models}

The comparison between analytical and numerical models was proceeded by testing the $3 \omega$ voltage fluctuations over a test case for chromel wire (diameter $13 \mu \mathrm{m}$ ) and carbon fiber (diameter $7 \mu \mathrm{m}$ ), all with a 1.5 mm length. An alternating current of $6 \mathrm{~mA}$ and $1 \mathrm{~mA}$ was assumed to pass through the chromel wire and carbon fiber respectively. The values of thermal properties were obtained from the literature or from the wire or fiber suppliers (Table 5 and Table 6). The computed values of $V_{3 \omega r m s}$ under vacuum, by using analytical and numerical (with $\mathrm{h}=0$ ) models iare compared in Fig. 2. It showed a good agreement between the $V_{3 \omega \mathrm{rms}}$ values. The difference lies mostly at lower frequencies $(f<0.1 \mathrm{~Hz})$. with a $V_{3 \omega r m s}$ value less than $1.6 \%$ of maximum $V_{3 \omega r m s}$. An analogous comparison between the two thermal models was also performed for the case of carbon fiber leading to similar results (discrepancy smaller than $1.6 \%$ of the maximum value of $V_{3 \omega \mathrm{rms}}$ ). Additionally, for each test, the validity condition of Eq. 4 for the analytical model is well checked with values of $\delta_{0}$ as $4 \times 10^{-4}$ for chromel and as $4 \times 10^{-2}$ for carbon fiber.

Fig 2 Numerical and analytical models comparison and effect of convective losses (13.6 $\mu \mathrm{m}$ chromel wire)

To demonstrate the influence of convective losses, one have chosen an approximate value of heat transfer coefficient using a correlation for natural air convection around a horizontal cylinder [17] with an assumption of max $1 \mathrm{~K}$ [9] increase in temperature. The radiation losses can be neglected as the criterion of Eq.8 is always respected for the chromel wire $\left(\delta_{l} \sim 0.025\right)$ and also carbon fibers $\left(\delta_{l} \sim 0.079\right)$. It can be observed from Fig 22 that the convective heat transfer leads to a huge drop of the $V_{3 \omega r m s}$ value. Fitting of the analytical model was performed on this case in order to quantify the effect of convective heat loss on the estimation of thermal conductivity and volumetric heat capacity. Table 3 shows that the thermal conductivity value is increased by $\sim 390 \%$ and the volumetric heat capacity value is decreased by $\sim 8 \%$ for chromel wire if one uses the analytical model which does not take into account heat losses. These biases become more prominent in case of carbon fiber (Table 3) as its thermal conductivity is smaller and also as the approximated heat transfer coefficient almost doubled due to the smaller diameter of carbon fiber. This shows the importance of using vacuum while performing measurements for 
the estimation of especially thermal conductivity of wire or fibers. For our experimental tests, we have chosen a secondary vacuum with a $10^{-6}$ mbar pressure resulting in an air thermal conductivity decrease of four orders of magnitude [18].

\begin{tabular}{lccc}
\hline Sample & Heat transfer coef. & \multicolumn{2}{c}{$\begin{array}{c}\text { Thermal properties } \\
\text { (Analytical model) }\end{array}$} \\
\hline \multirow{2}{*}{ Chromel wire } & 0 & $\mathrm{k}\left(\mathrm{Wm}^{-1} \mathrm{~K}^{-1}\right)$ & $\rho \mathrm{C}_{\mathrm{P}}\left(\mathrm{MJ} \mathrm{m}^{-3} \mathrm{~K}^{-1}\right)$ \\
\cline { 2 - 4 } & $\left.70 \mathrm{Wm}^{-2} \mathrm{~K}^{-1}\right)$ & 17 & 3.8 \\
Carbon fiber & 0 & 66 & 3.48 \\
& $1300^{*}$ & 10 & 1.3 \\
\hline
\end{tabular}

Table 3 Thermal conductivity and volumetric heat capacity estimated with and without convective losses and using the analytical model (Eq.7)

\section{Sensitivity Analysis}

For the design of new experiments for thermal properties measurement, it is very useful to perform sensitivity analysis of the measured quantities with respect to the unknown parameters. It allows to find the set of experimental conditions that will maximize the sensitivity coefficients and therefore will insure a greater accuracy of the estimated values. In our case, the sensitivity coefficients of the voltage $V_{3 \omega r m s}$ with respect to $k$ and $\rho C_{p}$ were studied as function of the frequency and also the sample length.

Fig 3. Sensitivity analysis of the $V_{3 \omega \mathrm{rms}}$ voltage to the (a) thermal conductivity and (b) volumetric heat capacity

As these sensitivity coefficients depend strongly on the sample length, one have considered their reduced expression $\left(X^{*}{ }_{m}\right)$ defined as [19]:

$X_{m}^{*}=m \frac{\partial V_{3 \omega r m s}}{\max \left(V_{3 \omega r m s}\right) \partial m}, \quad$ with $m=k, \rho C_{p}$

The reduced sensitivity coefficients for chromel wire and two types of PAN fibers were computed for sample lengths ranging from 0.5 to $3.5 \mathrm{~mm}$. The thermal conductivity and volumetric heat capacity values were obtained from the literature or from the supplier (Table 5 and Table 6). Fig 33 shows that the sensitivities of voltage $V_{3 \omega}$ rms with respect to $k$ and $\rho C_{p}$ are high in a particular frequency range depending on the sample length. Therefore if one considers a frequency range of 1 to $100 \mathrm{~Hz}$ (which provides reasonable measurement duration), the sample length should not exceed too much $1.5 \mathrm{~mm}$. 


\section{Experimental setup and samples}

The constant voltage source based circuit using a Wheatstone bridge has the disadvantage of the measurement of thermal properties for sample of only low electrical resistance, typically less than $400 \Omega$. As the electrical resistance of carbon fibers are higher (around $500 \Omega$ for a length of $1.5 \mathrm{~mm}$ ), therefore two differential amplifiers (AD624) with a constant current source (Keithley 6221) were used for the detection of the voltage signal in the sample $(R)$ and the variable resistance $\left(R_{V}\right)$ as shown in Fig. 4(a). The signal from these two resistances were fed to the inputs A and B of the Lock in Amplifier (LIA Ametek 7265). The LIA was set to the configuration of A-B, a subtraction mode of the signal from the two AD624. The LIA was first set to $1 \omega$ detection mode, which needs to be equal to 0 at each frequency involving $R_{V}=R$ and ultimately led to the cancellation of $1 \omega$ signal. The variable additional resistance was pure resistance with no significant temperature increase. Then the LIA was set to the $3 \omega$ detection mode and thus the A-B mode provides the $3 \omega$ signal only through the sample.

Fig 4 (a) Electrical setup for $3 \omega$ measurement (b) Sample holder (c) Specimen attached to the electrode with silver paste (d) Cross section of the PAN carbon fiber(FT300B) obtained from scanning electron microscopy

The thermal conductivity and volumetric heat capacity were estimated for the chromel wire and two PAN carbon fibers from Toray Industries, FT300B and FT800H. Single fiber/wire was placed between two copper plates as shown in Fig. 4(b) and (c) and covered carefully with silver paste and given sufficient time for the silver paste to dry Fig. 4 (c). The copper electrodes are acting as both heat sinks and electrical connectors. An accurate measurement of the parameters such as sample length and diameter, involved in the analytical and the numerical model was necessary. A Scanning Electron Microscope and a high-resolution optical camera were used to have an exact measurement of the sample diameter and length respectively. For this paper, the length of each sample was chosen in such a way that the working range frequency is $1-100 \mathrm{~Hz}$ thanks to the previous sensitivity analysis, making the $3 \omega$ measurements quite fast ( $30 \mathrm{~min}$ for 25 frequency values). Table 4 presents the lengths of the chromel wire and carbon fibers used for performing the $3 \omega$ experiments. Later the sample was then placed in a chamber that can work as a secondary vacuum $\left(10^{-6} \mathrm{mbar}\right)$ at room temperature. In order to show the influence of convective heat loss in the parameter estimation by the analytical model, the experiments were also performed at atmospheric pressure $\left(10^{3}\right.$ mbar $)$ for chromel wire.

\begin{tabular}{cccc}
\hline \multicolumn{2}{c}{ Type of fiber } & Diameter $(\boldsymbol{\mu m})$ & Length $(\mathbf{m m})$ \\
\hline \multirow{2}{*}{ FT300B } & Sample 1 & $6.95-7.23$ & 1.24 \\
& Sample 2 & $6.69-7.01$ & 1.34 \\
& Sample 3 & $7.32-7.61$ & 1.53 \\
FT800H & Sample 4 & $6.99-7.62$ & 1.76 \\
Chromel & & $5.45-5.79$ & 1.56 \\
\hline
\end{tabular}

Table 4 Dimension of the samples used for $3 \omega$ measurement

Despite the use of instruments with high accuracy, there are few uncertainties on known parameters such as length, diameter, current and etc. The model for the estimation of the overall uncertainty on the thermal conductivity and volumetric heat capacity through transient measurements was presented by Milosevic[20]. 


$$
S_{\text {final }}=\left[X^{T} W X\right]^{-1} \text { with } W=\left[\sigma_{V_{3 \omega r m s}}^{2}+\sum_{p}\left(\sigma_{m_{p}} \frac{\partial V_{3 \omega r m s}}{\partial m_{p}}\right)^{2}\right]^{-1}
$$

where $X$ is the sensitivity coefficient matrix, $W$ is the variance covariance matrix, $\sigma_{V_{3} \omega r m s}$ is the variance of measured $V_{3 \omega r m s}, \sigma_{m_{p}}$ is the variance of the known parameters $p, S_{f i n a l}$ is the matrix of variance and covariance.

The values of $\sigma_{m_{p}}$ can be estimated with the errors on the known parameters during measurements of $V_{3 \omega r m s}$ for chromel wire represented as $e_{I}=0.1 \%, e_{R}=0.5 \%, e_{\alpha_{e}}=0.5 \%, e_{D}=0.2 \%, e_{L}=2.8 \%$. During the measurements for carbon fiber, the error is similar to the chromel except in case of the diameter for which $e_{D}=5.1 \%$ predicted from Table 4. The diagonal terms of $S_{\text {final }}$ represent the variances $\sigma_{m}{ }^{2}$ of the estimated parameters $m\left(k\right.$ or $\left.\rho C_{p}\right)$ including the effect of the uncertainty of the known parameters. For a $95 \%$ confidence band, the relative uncertainty $e_{m}$ on parameter $m$ is obtained using $e_{m}=1.96 \sigma_{m} / m$.

\section{Experimental Results}

\subsection{Chromel wire}

The validation of the experimental setup was achieved by choosing a wire with known properties. Therefore a $13.6 \mu \mathrm{m}$ diameter chromel wire was tested. Its diameter was checked to be fairly constant with perfectly circular cross-section over the length. Fig. 5 shows the experimental test performed in vacuum and atmospheric condition for a frequency range of 1 to $100 \mathrm{~Hz}$. Both experimental data were fitted by the analytical model to determine the thermal conductivity and volumetric heat capacity of the chromel wire. Table 5 shows the estimated values of the thermal conductivity and volumetric heat capacity for the chromel wire by using $3 \omega$ method. The experiments conducted under vacuum showed very good agreement of the measured values for thermal conductivity and volumetric heat capacity with the reference values from the literature. The overall uncertainties in the measurement of thermal conductivity and volumetric heat capacity for chromel are $\sim 2.9 \%$ and $\sim 1.3 \%$ respectively (section 6). As expected from our initial remark with the influence of convective loss, the thermal conductivity estimated from the experiments performed under the atmospheric condition is very unrealistic and with a discrepancy of $\sim 390 \%$ from the reference value [21]. In case of fitting by the numerical model for experiments in an atmospheric condition, the heat transfer coefficient was approximated to be ranging from $450-740 \mathrm{Wm}^{-2} \mathrm{~K}^{-1}$ for a temperature rise from 0.1 to $2 \mathrm{~K}$ and using the correlations presented by Churchill and Chu [17] and also Morgan [25] which are valid for the obtained Rayleigh number during our experiments. The impact of the range of heat transfer coefficient is shown in the estimation of the thermal properties (Table 5) by the numerical model leading the estimated thermal conductivity not reliable under atmospheric condition. In addition, one should note that the used correlations were established for steady state regimes and not periodic ones.

Fig 5 Measured $(\bullet)$ and fitted $(-) \mathrm{V}_{3 \omega}$ voltages for chromel wire with vacuum and atmospheric conditions 


\begin{tabular}{ccccc}
\hline Thermal Properties & & \multicolumn{2}{c}{ Measured } & Reference[21][22] \\
\hline & Vacuum & Analytical Model & Numerical Model & \\
& & 18.15 & 18.27 & 17.3 \\
$\mathrm{k}\left(\mathrm{Wm}^{-1} \mathrm{~K}^{-1}\right)$ & Atmospheric & 70.15 & $18.51-25.82 *$ & 3.81 \\
& Vacuum & 3.68 & $3.81-3.83 *$ & 3.85 \\
\hline
\end{tabular}

Table 5 Thermal properties measured for chromel wire by fitting with the analytical model under vacuum and atmospheric condition (* this range is due to the range of $h$ value from the correlations [17] and [25] and also due to a 0.1 to $2 \mathrm{~K}$ temperature rise)

\subsection{Carbon fibers}

The $3 \omega$ method was then used to estimate the thermal properties for the two PAN carbon fibers. Fig. 6(a) shows the experimental $3 \omega$ voltage fluctuations with a 1 to $100 \mathrm{~Hz}$ frequency range performed in a vacuum chamber. The thermal conductivity and volumetric heat capacity were measured for the four samples of FT300B with different lengths by fitting with the analytical model. Fiber FT800HB was tested for single length of the sample of $1.56 \mathrm{~mm}$ (Fig. 6(b)). The measured values of thermal conductivity and volumetric heat capacity for the carbon fibers are presented in Table 6 and compared with the data provided by the fiber supplier (Toray Industries). The uncertainties in the measurement of thermal conductivity and volumetric heat capacity are $\sim 8.1 \%$ and $\sim 4.9 \%$ respectively for these carbon fibers, as estimated from the uncertainty analysis shown in section 6.

Fig 6 Measured (•) and fitted (-) $\mathrm{V}_{3 \omega}$ voltages for (a) FT300B carbon fibers with multiple sample lengths, (b) FT800HB carbon fiber with a $1.56 \mathrm{~mm}$ sample length

\begin{tabular}{|c|c|c|c|c|c|c|c|}
\hline \multirow[b]{2}{*}{$\begin{array}{c}\text { Thermal } \\
\text { Properties }\end{array}$} & \multicolumn{4}{|c|}{ FT300B } & \multirow{2}{*}{$\begin{array}{c}\begin{array}{c}\text { Reference } \\
{[23]}\end{array} \\
\text { Supplier } \\
\text { datasheet }\end{array}$} & \multirow[t]{2}{*}{ FT800H } & \multirow{2}{*}{$\begin{array}{c}\text { Reference } \\
{[24]} \\
\text { Supplier } \\
\text { datasheet }\end{array}$} \\
\hline & Sample 1 & Sample 2 & Sample 3 & Sample 4 & & & \\
\hline $\mathrm{k}\left(\mathrm{Wm}^{-1} \mathrm{~K}^{-1}\right)$ & 10.11 & 10.03 & 10.17 & 10.28 & 10.47 & 34.88 & 35.13 \\
\hline$\rho C_{P}\left(M^{-3} m^{-3} K^{-1}\right)$ & 1.35 & 1.38 & 1.37 & 1.37 & 1.39 & 1.37 & 1.36 \\
\hline
\end{tabular}

Table 6 Thermal properties estimation for carbon fibers

The experiments performed under atmospheric condition could give an approximate range of thermal property with the condition of proper estimation of theoretical heat transfer coefficient. This is impossible to achieve in case of carbon fiber due to the complex cross section as shown from the SEM image (Fig. 4(d)) and the reliability of the correlation for the estimation of heat transfer coefficient. Although the numerical model could be used for the estimation of heat transfer coefficient along with the thermal conductivity and volumetric heat capacity for an experiment performed under atmospheric condition, but it was observed that the thermal conductivity and heat transfer coefficient are highly correlated parameters, making impossible the correct estimation of these parameters simultaneously. 


\subsection{Effect of thermal contact resistance between carbon fiber and copper electrodes}

The analytical and numerical model works with an assumption of perfect thermal contact between the sample and copper electrodes, which is impossible to achieve in practice. Therefore an estimation of thermal contact resistance was done from the measured values of thermal conductivity with different lengths of the sample by the calculation of the overall thermal resistance (L/k) for each length. Fig. 7 shows the evolution of thermal resistance with length for the fiber FT300B.

Fig 7 Thermal resistance vs length for FT300B

The measured thermal resistances can be fitted with linear regression. The y intercept of this plot gives the thermal contact resistance when the fiber length is 0 . The obtained value $\left(8.8310^{-6} \mathrm{~m}^{2} \mathrm{KW}^{-1}\right)$ can be compared to the intrinsic thermal resistance of the fibers $\left(\mathrm{L} / \mathrm{k} \sim 10^{-4} \mathrm{~m}^{2} \mathrm{KW}^{-1}\right)$. The overall effect of the thermal contact resistance for the $3 \omega$ method is, therefore less than $7 \%$ of the thermal resistance $(\mathrm{L} / \mathrm{k})$. The inverse of the slope of the linear regression provides the final value of the thermal conductivity of the FT300B carbon fiber which is equal to 10.82 $\mathrm{Wm}^{-1} \mathrm{~K}^{-1}$.

\section{Conclusions}

The analytical model for fiber developed by $\mathrm{Lu}$ [9] was found to be accurate enough to estimate the thermal conductivity and volumetric heat capacity of small metallic wire and carbon fibers. The importance of experimenting only under vacuum condition was proven by quantifying the influence of convective loss on the estimation of thermal conductivity using this analytical model and a numerical model able to take into account the heat losses. The effect on thermal conductivity is very high, especially for sample of low thermal conductivity. The volumetric heat capacity turned out to be not so affected by convective heat losses. A detailed sensitivity analysis for both thermal conductivity and volumetric heat capacity was done for getting results with higher accuracy. It indeed enabled the choice of an adequate frequency range and sample length. Based on the experimental configuration of differential amplifiers with a constant current source, the results for small chromel wire and carbon fibers obtained under vacuum showed high precision with respect to the reference values. The higher uncertainty in the measurement for carbon fibers is mainly due to the discrepancy of the diameter through the length of samples. The sum of the two thermal contact resistances at both fiber ends was also estimated to be less than $7 \%$ of the overall thermal resistance of the sample thus the influence on the measurement of thermal conductivity is small. Finally, the adaptation of the $3 \omega$ method to the measurement of the thermal properties of carbon fibers presented here is proved to be efficient and reliable. Furthermore, the uncertainties were thoroughly calculated and provided an acceptable interval of confidence. Further work will be dedicated to the measurement of the radial thermal conductivity of carbon fibers.

Acknowledgements: The authors would like to thanks J. Aubril for the discussions and quality of his technical realizations. 


\section{References}

1. Sweeting RD, Liu XL. Measurement of thermal conductivity for fibre-reinforced composites. Compos Part A Appl Sci Manuf. 2004;35:933-8.

2. Manocha LM, Warrier A, Manocha S, Sathiyamoorthy D, Banerjee S. Thermophysical properties of densified pitch based carbon/carbon materials-I. Bidirectional composites. Carbon N Y. 2006;44:488-95.

3. Siddiqui MOR, Sun D. Development of Experimental Setup for Measuring the Thermal Conductivity of Textiles. Cloth Text Res J. 2018;36:215-30.

4. Gallego NC, Edie DD, Ntasin LN, Ervin VJ. Modeling the thermal conductivity of carbon fibers. Carbon N Y. 2000;38:1003-10.

5. Craddock JD, Burgess JJ, Edrington SE, Weisenberger MC. Method for Direct Measurement of On-Axis Carbon Fiber Thermal Diffusivity Using the Laser Flash Technique. J Therm Sci Eng Appl [Internet]. 2016;9:014502.

6. Yamane T, Katayama SI, Todoki M, Hatta I. Thermal diffusivity measurement of single fibers by an ac calorimetric method. J Appl Phys. 1996;80:4358-65.

7. Wang JL, Gu M, Zhang X, Song Y. Thermal conductivity measurement of an individual fibre using a T type probe method. J Phys D Appl Phys. 2009;42.

8. Pradere C, Batsale JC, Goyhénèche JM, Pailler R, Dilhaire S. Thermal properties of carbon fibers at very high temperature. Carbon N Y. 2009;47:737-43.

9. Lu L, Yi W, Zhang DL. $3 \omega$ method for specific heat and thermal conductivity measurements. Rev Sci Instrum. 2001;72:2996-3003.

10. Liang J. Experimental measurement and modeling of thermal conductivities of carbon fibers and their composites modified with carbon nanofibers. $\mathrm{PhD}$ thesis University of Oklahoma, 2014. https://shareok.org/handle/11244/10398?show=full

11. Schiffres SN, Malen JA. Improved 3-omega measurement of thermal conductivity in liquid, gases, and powders using a metal-coated optical fiber. Rev Sci Instrum. 2011;82.

12. Gauthier S, Giani A, Combette P. Gas thermal conductivity measurement using the three-omega method. Sensors Actuators, A Phys [Internet]. Elsevier B.V.; 2013;195:50-5. http://dx.doi.org/10.1016/j.sna.2012.12.032

13. Wang ZL, Tang DW, Zhang WG. Simultaneous measurements of the thermal conductivity, thermal capacity and thermal diffusivity of an individual carbon fibre. J Phys D Appl Phys. 2007;40:4686-90.

14. Pradère C, Goyhénèche JM, Batsale JC, Dilhaire S, Pailler R. Thermal diffusivity measurements on a single fiber with microscale diameter at very high temperature. Int J Therm Sci. 2006;45:443-51.

15. Xing C, Jensen C, Munro T, White B, Ban H, Chirtoc M. Accurate thermal property measurement of fine fibers by the 3-omega technique. Appl Therm Eng. 2014;73:315-22.

16. Hou J, Wang X, Vellelacheruvu P, Guo J, Liu C, Cheng HM. Thermal characterization of single-wall carbon nanotube bundles using the self-heating $3 \omega$ technique. J Appl Phys. 2006;100.

17. Boetcher SKS. Natural convection transfer from horizontal cylinders. SpringerBriefs Appl Sci Technol. $2014 ; 23-42$.

18. Ediss GA. Effect of Vacuum Pressure on the Thermal Loading of the ALMA Cryostat. Natl Radio Astron Obs ALMA. 2006;Memo 554:1-3.http://legacy.nrao.edu/alma/memos/html-memos/alma554/memo554.pdf

19. Chapelle E, Garnier B, Bourouga B. Interfacial thermal resistance measurement between metallic wire and polymer in polymer matrix composites. Int J Therm Sci [Internet]. Elsevier Masson SAS; 2009;48:2221-7. 
http://dx.doi.org/10.1016/j.ijthermalsci.2009.05.001

20. Milošević ND, Raynaud M, Maglić KD. Estimation of thermal contact resistance between the materials of double-layer sample using the laser flash method. Inverse Probl Eng. 2002;10:85-103.

21. Sundqvist B. Thermal diffusivity and thermal conductivity of Chromel, Alumel, and Constantan in the range 100-450 K. J Appl Phys. 1992;72:539-45.

22. Omega Engineering, Physical Properties of Thermo-element Materials, Available online https://www.omega.com/techref/pdf/z016.pdf.;

23. Data sheet Torayca T300B, Available online http://www.toraycfa.com/pdfs/T300DataSheet.pdf..

24. Data sheet Torayca T800H, Available online https://www.toraycma.com/file_viewer.php?id=4463.

25. Morgan V. The overall convective heat transfer from smooth circular cylinders, Adv Heat Transf 11. 1975; 199-264. doi:10.1016/S0065-2717(08)70075-3 


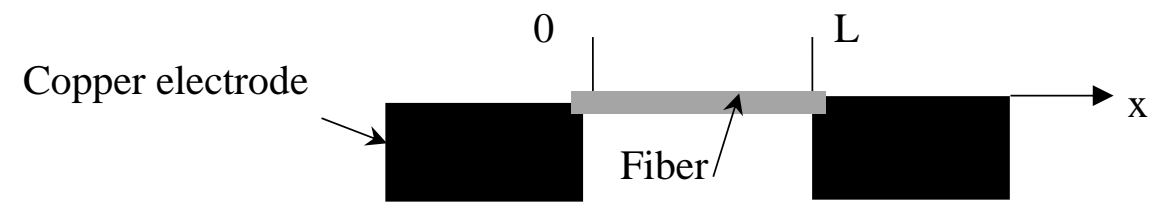

Fig 1

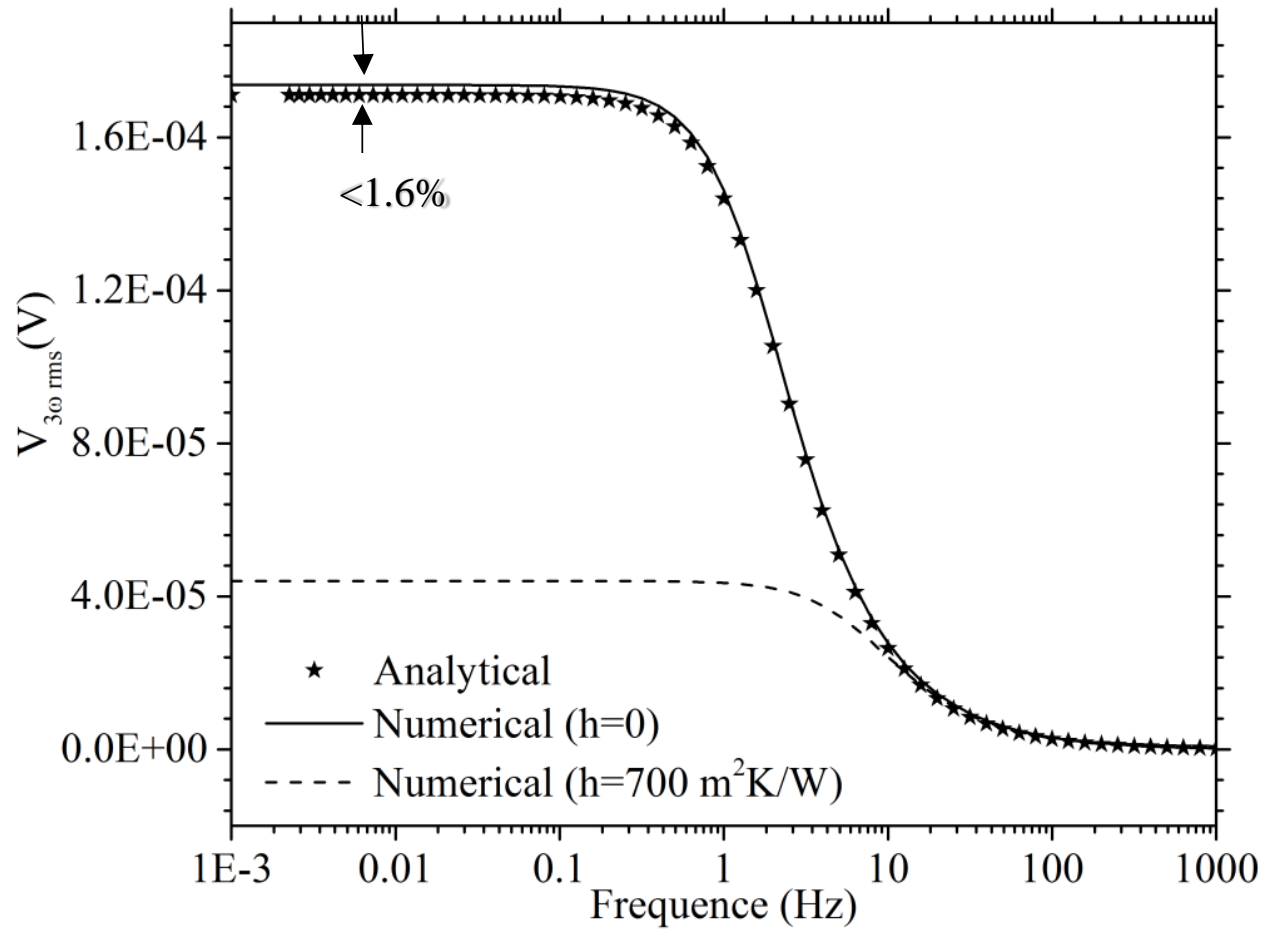

Fig 2 


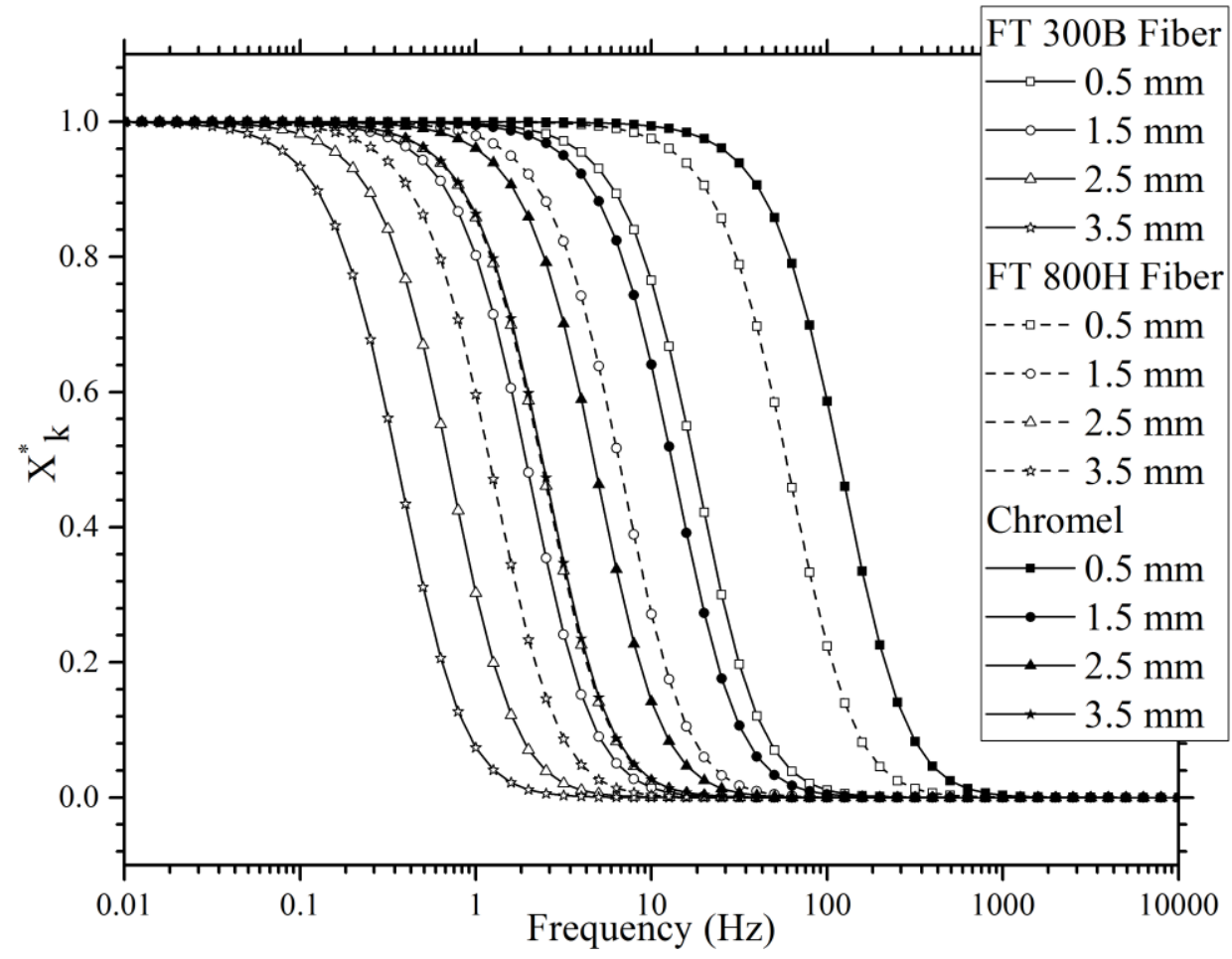

Fig 3 (a)

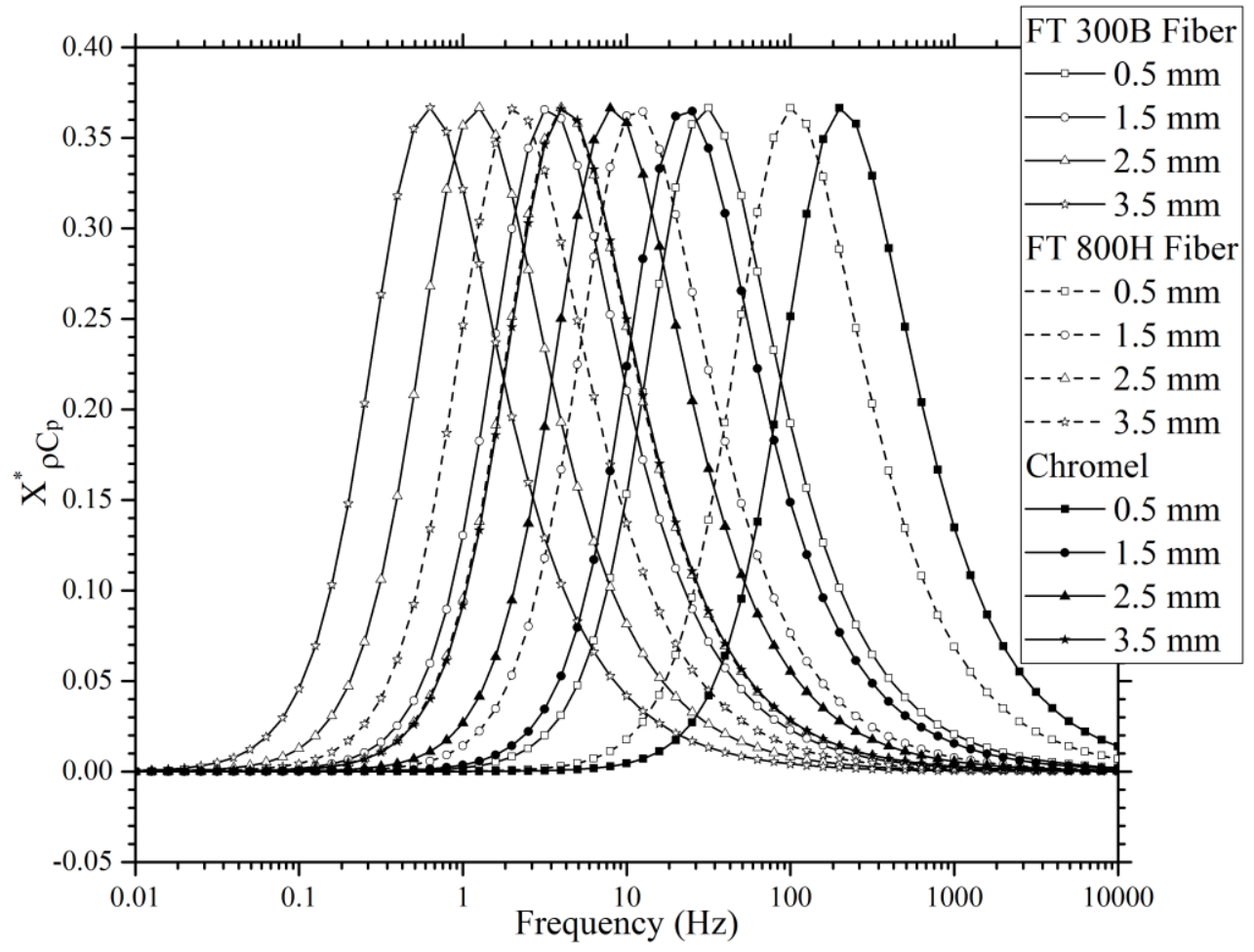

Fig 3 (b) 


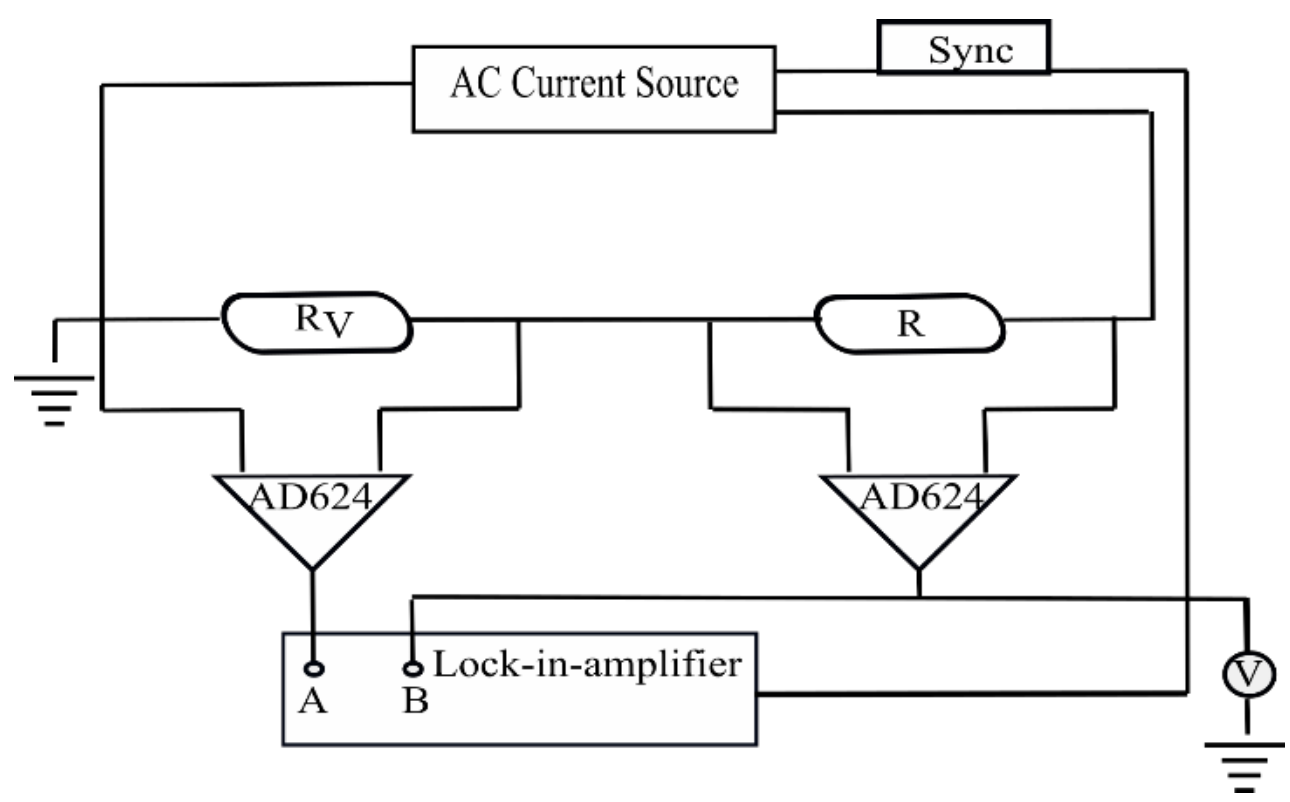

Fig 4 (a)

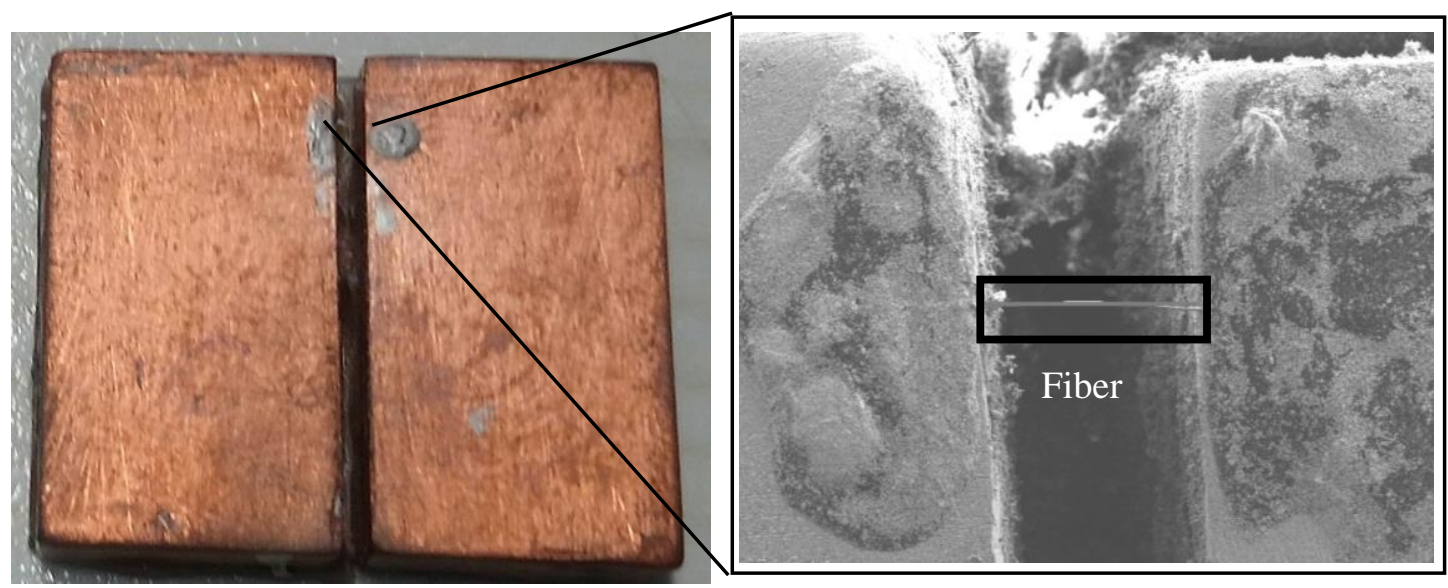

Fig 4 (b)

Fig 4 (c)

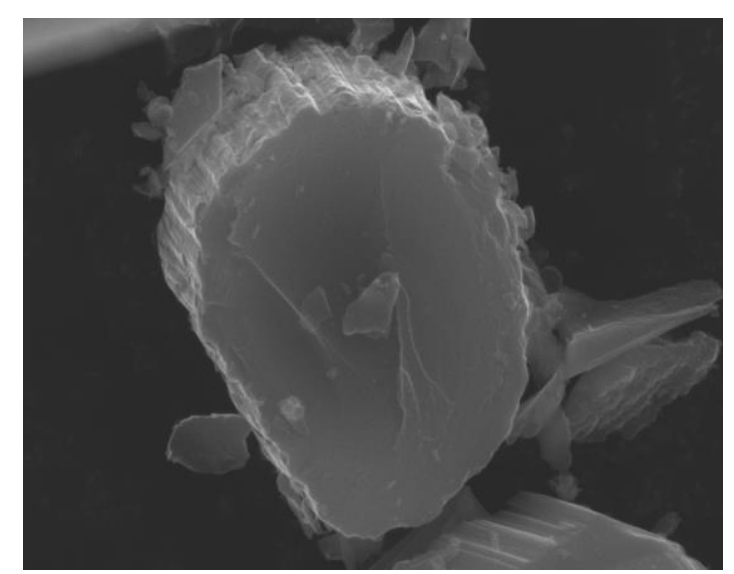

Fig 4 (d) 


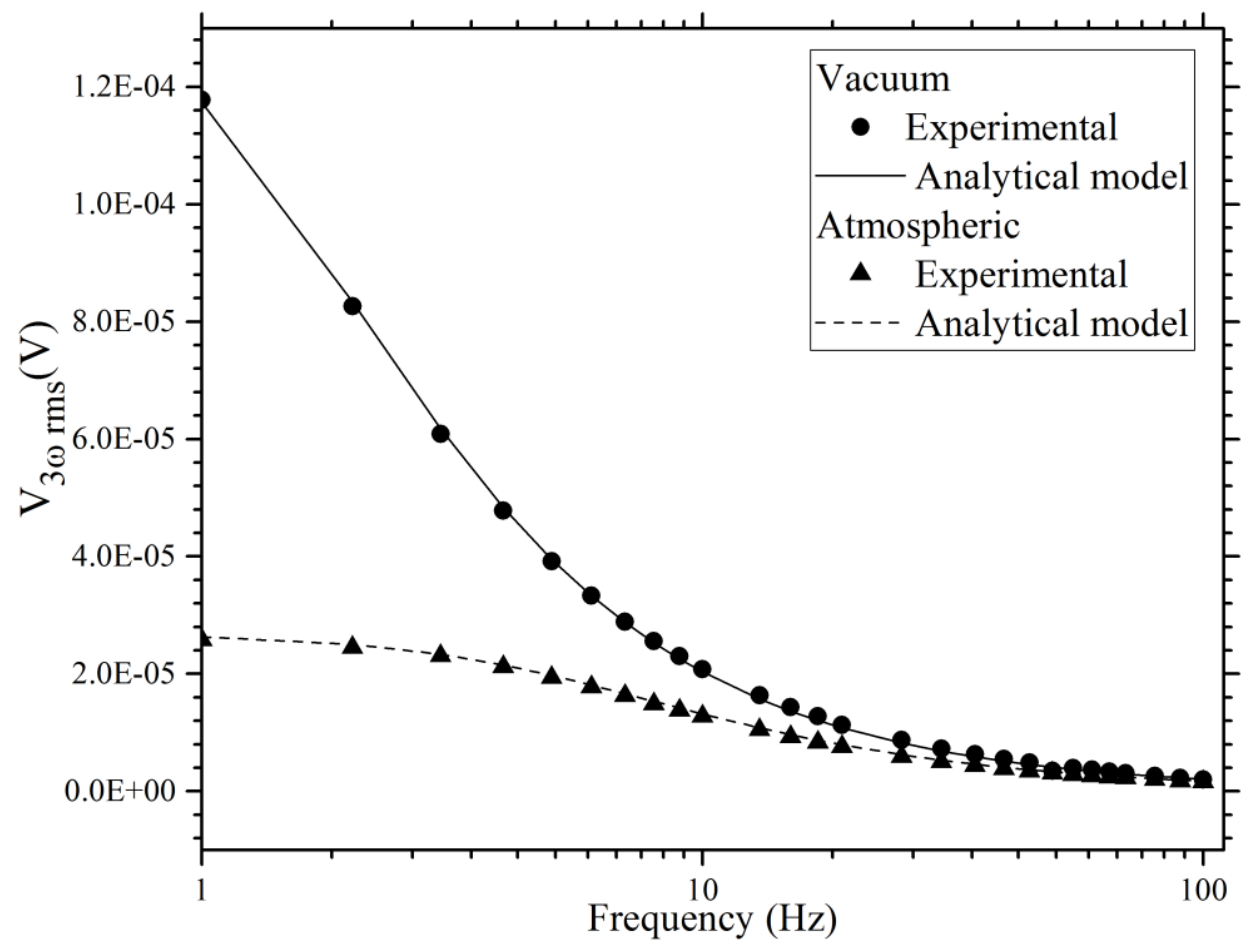

Fig 5

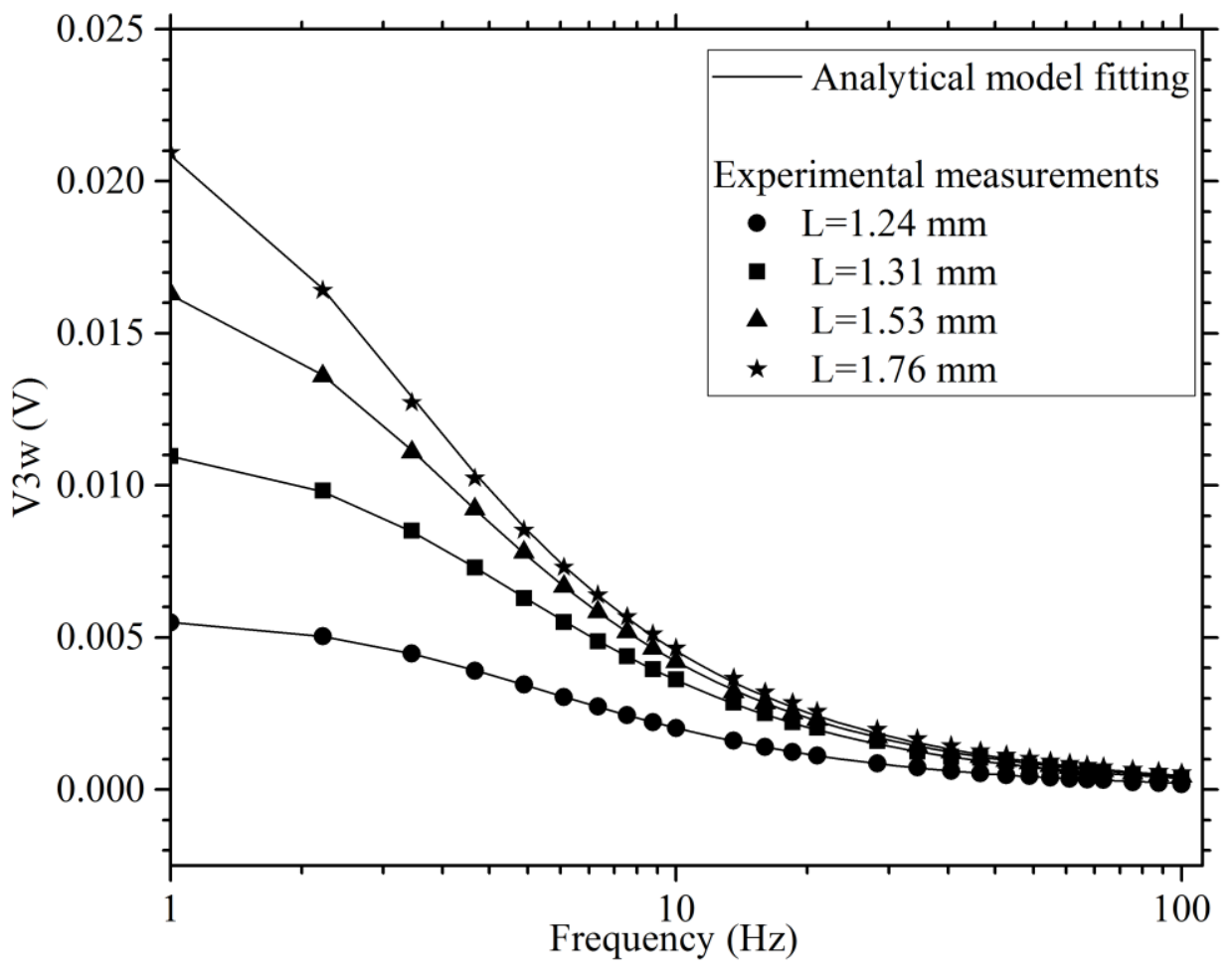

Fig 6 (a) 


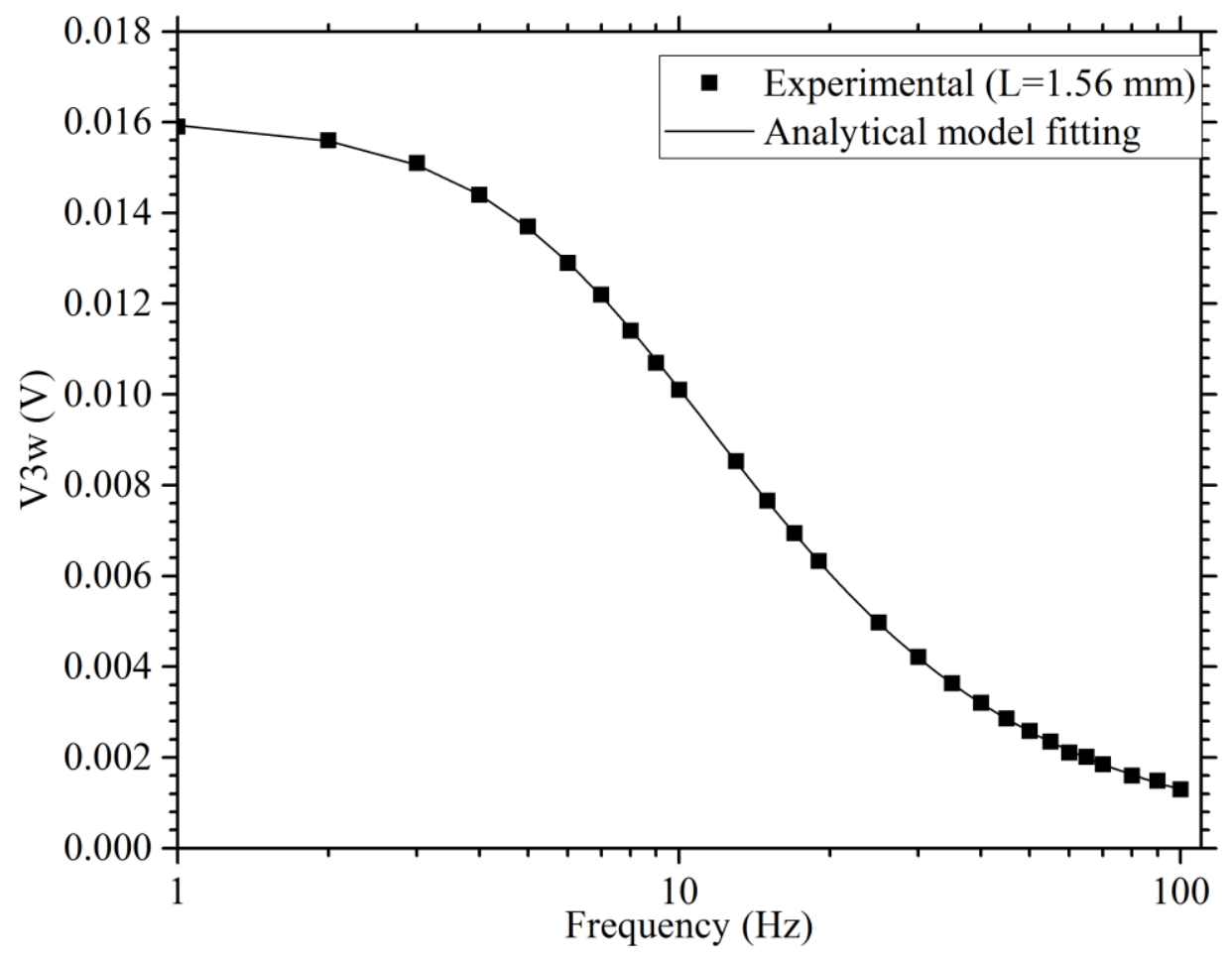

Fig 6 (b)

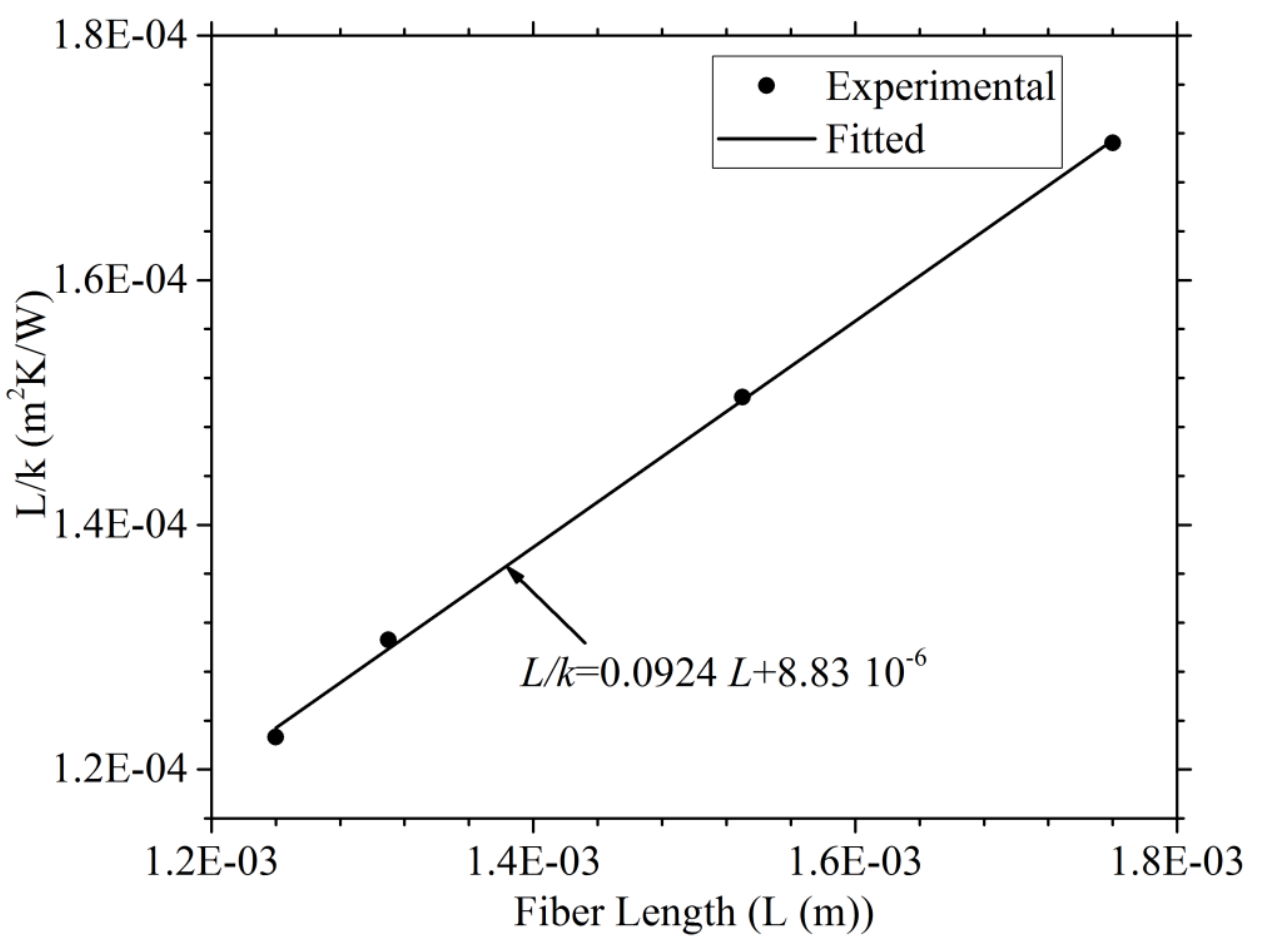

Fig 7 